\title{
Magnetic Field Configuration Around Large Flux Ropes
}

\author{
E. Romashets ${ }^{1}$ and M. Vandas ${ }^{2}$ \\ ${ }^{1}$ IZMIRAN, Troitsk, 149190, Russia \\ email: romash@izmiran.rssi.ru \\ ${ }^{2}$ Astronomical Institute, 14131 Praha 4, Czech Republic \\ email: vandas@ig.cas.cz
}

\begin{abstract}
An analytical method is used to model a magnetic field distribution in the vicinity of a large interplanetary or solar flux rope. The field is a sum of the pre-existing one and an additional current-free part. An example using real data is shown.
\end{abstract}

Keywords. Sun: magnetic fields, Sun: coronal mass ejections (CMEs), methods: analytical.

\section{Introduction}

It is widely accepted that magnetic flux ropes reside in the solar atmosphere and that some of them erupt and propagate in the interplanetary space. In the present paper we model a magnetic field $\mathbf{B}^{\text {tot }}$ around a flux rope. We start from a pre-existing ambient (background) field $\mathbf{B}^{\mathrm{amb}}$ and add an additional potential field $\mathbf{B}^{\text {add }}$. The rope is assumed to have a toroidal shape with a major radius $R_{0}$. Its minor radius $r_{0}$ may vary, depending on an azimuthal angle $\varphi$ along the circular toroid's axis (with the radius $R_{0}$ ).

In toroidal coordinates, $\mu, \eta$, and $\varphi$ (Vandas, Romashets \& Watari 2003) a boundary of the toroidal flux rope is determined by $\mu_{0}, \cosh \mu_{0}(\varphi)=R_{0} / r_{0}(\varphi)$. The exterior of the flux rope has $\mu>\mu_{0}$.

The field $\mathbf{B}^{\text {tot }}$ must have zero normal components at the flux rope boundary, i.e.,

$$
\left.\left(\mathbf{B}^{\text {tot }} \cdot \mathbf{n}\right)\right|_{\mu=\mu_{0}}=0
$$

must hold for all normal vectors $\mathbf{n}$ to the rope boundary. In order to fulfill the condition (1.1), an additional field $\mathbf{B}^{\text {add }}$ is added to the background field so the resulting external field is $\mathbf{B}^{\text {tot }}=\mathbf{B}^{\mathrm{amb}}+\mathbf{B}^{\text {add }}$. The additional field is supposed to be potential and vanishing at large distances from the flux rope.

According to Romashets \& Vandas (2004), the components of the resulting field are

$$
\begin{aligned}
B_{\mu}^{\mathrm{tot}} & =B_{\mu}^{\mathrm{amb}}+B_{0} \sinh \mu \sqrt{\cosh \mu-\cos \eta} \sum\left[\frac{1}{2} P_{n-1 / 2}^{m}(\cosh \mu)\right. \\
& \left.+(\cosh \mu-\cos \eta) P_{n-1 / 2}^{m \prime}(\cosh \mu)\right] \\
& \times\left(\alpha_{n}^{m} \cos n \eta \cos m \varphi+\beta_{n}^{m} \cos n \eta \sin m \varphi+\gamma_{n}^{m} \sin n \eta \cos m \varphi+\delta_{n}^{m} \sin n \eta \sin m \varphi\right), \\
B_{\eta}^{\mathrm{tot}} & =B_{\eta}^{\mathrm{amb}}+B_{0} \sqrt{\cosh \mu-\cos \eta} \sum P_{n-1 / 2}^{m}(\cosh \mu)\left[\frac { 1 } { 2 } \operatorname { s i n } \eta \left(\alpha_{n}^{m} \cos n \eta \cos m \varphi\right.\right. \\
& \left.+\beta_{n}^{m} \cos n \eta \sin m \varphi+\gamma_{n}^{m} \sin n \eta \cos m \varphi+\delta_{n}^{m} \sin n \eta \sin m \varphi\right)+n(\cosh \mu-\cos \eta) \\
& \left.\times\left(-\alpha_{n}^{m} \sin n \eta \cos m \varphi-\beta_{n}^{m} \sin n \eta \sin m \varphi+\gamma_{n}^{m} \cos n \eta \cos m \varphi+\delta_{n}^{m} \cos n \eta \sin m \varphi\right)\right],
\end{aligned}
$$




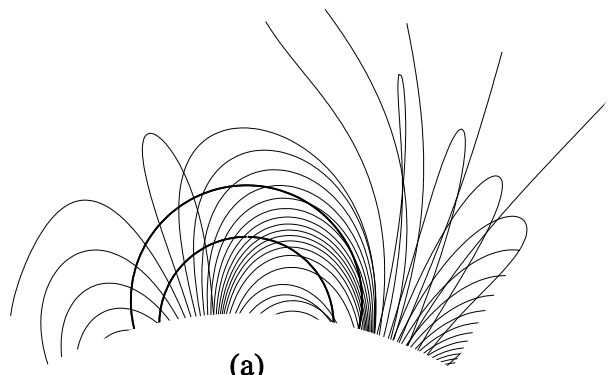

(a)

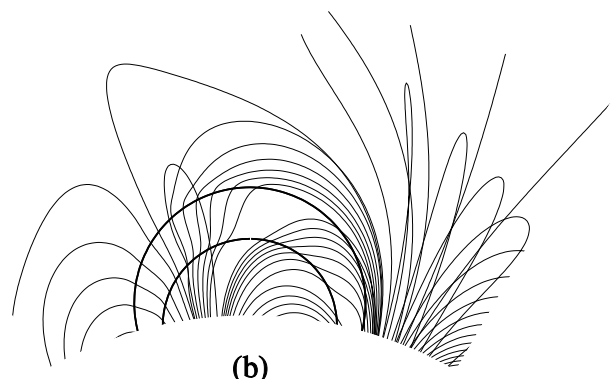

(b)

Figure 1. (a) Magnetic field configuration in the solar atmosphere based on real photospheric observations. An assumed loop-like flux rope is drawn only for comparison with figure (b) showing a modified field when this flux rope is taken into account.

$$
\begin{aligned}
B_{\varphi}^{\mathrm{tot}} & =B_{\varphi}^{\mathrm{amb}}+B_{0} \frac{(\cosh \mu-\cos \eta)^{3 / 2}}{\sinh \mu} \sum m P_{n-1 / 2}^{m}(\cosh \mu) \\
& \times\left(-\alpha_{n}^{m} \cos n \eta \sin m \varphi+\beta_{n}^{m} \cos n \eta \cos m \varphi-\gamma_{n}^{m} \sin n \eta \sin m \varphi+\delta_{n}^{m} \sin n \eta \cos m \varphi\right) .
\end{aligned}
$$

$P_{n-1 / 2}^{m}$ is a Legendre function, its derivative by an argument is denoted by a prime. Coefficients $\alpha_{n}^{m}, \beta_{n}^{m}, \gamma_{n}^{m}$, and $\delta_{n}^{m}$ are found numerically in order (1.1) to be approximately satisfied. Summations in (1.2)-(1.4) are theoretically over $n=0, \infty$ and $m=0, \infty$, but for numerical calculations they are cut at some suitable $n=N$ and $m=M$.

\section{An example of an external field}

Romashets \& Vandas (2004) showed modified fields for two simple cases of ambient preexisting fields, uniform and radial. We present a much more complex case. Figure 1a shows magnetic field lines in the solar atmosphere based on real photospheric observations. Coefficients provided by the Wilcox Solar Observatory for an ad-hoc chosen period were used to construct this ambient potential field $\left(\mathbf{B}^{\mathrm{amb}}\right)$; see, e.g., Hoeksema \& Scherrer (1986). Let us introduce a flux rope into the field (thick lines). The flux rope is anchored in the photosphere. Two groups of field lines are displayed. The lines of the first group do not intersect the flux rope; these are the lines which do not enter into the silhouette of the rope in Figure 1a. The second group are remaining lines drawn in Figure 1a; all they cross near the central axial line of the rope. Figure $1 \mathrm{~b}$ shows magnetic field lines after the modification (field $\mathbf{B}^{\text {tot }}$ ). No field line penetrates the flux rope. The field lines of the first group are nearly not or only slightly modified, in dependence on their distance from the flux rope. The field lines of the second group drape around the flux rope.

\section{Conclusions}

A new method to model magnetic field configurations outside solar flux ropes has been applied to real data. Such configuration can be used for initialization of MHD simulations for space weather events.

\section{Acknowledgements}

This work was supported by INTAS grant 03-51-6206, AV ČR project S1003006, MŠMT ČR project ME501, and RFBR grant 03-02-16340.

\section{References}

Hoeksema, J. T. \& Scherrer, P. H. 1986, Sol. Phys. 105, 205

Vandas, M., Romashets, E. P. \& Watari, S. 2003, Astron. Astrophys. 412, 281

Romashets, E. P. \& Vandas, M. 2004, in: A. V. Stepanov et al. (eds.), Proc. IAU Symp. 223 'Multi-Wavelength Investigations of Solar Activity', in press 